\title{
Negative thermophoresis in concentric carbon nanotube
}

\section{nanodevices: Supporting Information}

\author{
Jiantao Leng ${ }^{1}$, Zhengrong Guo ${ }^{1}$, Hongwei Zhang ${ }^{1}$, Tienchong Chang ${ }^{1,}$, Xingming Guo ${ }^{1}$, \\ Huajian $\mathbf{G a o}^{2}$ \\ ${ }^{1}$ Shanghai Institute of Applied Mathematics and Mechanics, Shanghai Key Laboratory of Mechanics in \\ Energy Engineering, Shanghai University, Shanghai 200072, People’s Republic of China \\ ${ }^{2}$ School of Engineering, Brown University, Providence, RI 02912, USA
}

By establishing an equivalent thermal circuit, we develop a theoretical model for the observed geometry dependent thermophoretic force in the carbon nanotube nanodevice under consideration. The details of derivation are presented in the following.

Temperature gradient. Thermophoretic motion in the considered device is indeed induced by the inner tube temperature gradient caused by the temperature difference between two outer tubes. The heat flow from the high temperature outer tube, through the inner tube, to the low temperature outer tube provides the driving force for the inner tube motion. It is thus important to know the thermal gradient on the inner tube.

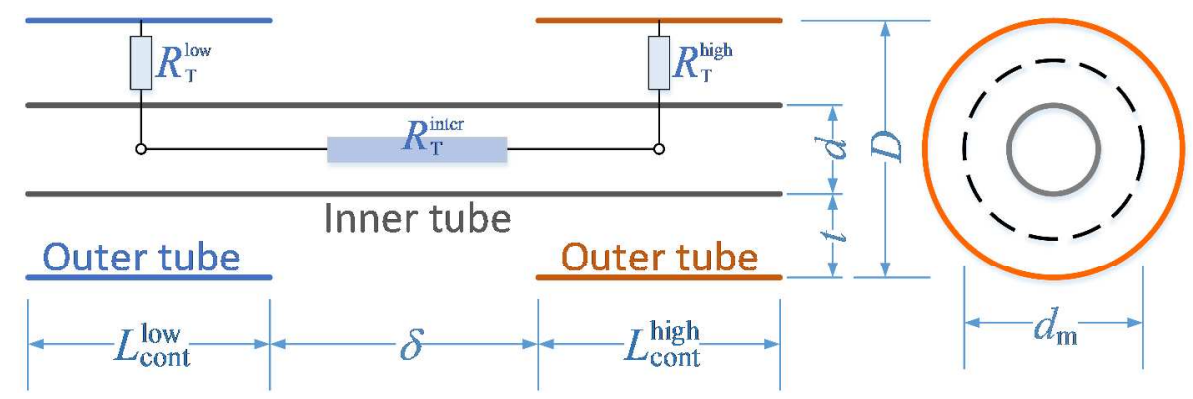

FIGURE S1. Equivalent circuit of thermal transport in the considered device.

\footnotetext{
*Email address: tchang@staff.shu.edu.cn.
} 
Consider the equivalent circuit, shown in Figure S1, of the devices under consideration. The temperature gradient on the inner tube induced by the temperature difference between the two outer tubes can be calculated from

$$
\nabla T=\frac{R_{\mathrm{T}}^{\text {iner }}}{R_{\mathrm{T}}^{\text {low }}+R_{\mathrm{T}}^{\text {inner }}+R_{\mathrm{T}}^{\text {high }}} \frac{\Delta T}{\delta+\left(L_{\text {cont }}^{\text {low }}+L_{\text {cont }}^{\text {high }}\right) / 2},
$$

where $R_{\mathrm{T}}^{\text {high }}, R_{\mathrm{T}}^{\text {inner }}$, and $R_{\mathrm{T}}^{\text {low }}$ are the thermal resistances when heat flows through the high temperature interlayer part, the inner tube part, and the low temperature interlayer part; $L_{\text {cont }}^{\text {low }}$ and $L_{\text {cont }}^{\text {high }}$ are the contact lengths between the two tubes at the low temperature side and the high temperature side, respectively; and $\delta$ is the distance between the neighboring ends of the outer tubes.

The interlayer thermal resistance is reversely dependent on the interlayer heat transfer coefficient and the interlayer contact area, i.e.,

$$
R_{\mathrm{T}}^{\text {low }}=\frac{1}{\pi d_{\mathrm{M}} L_{\text {cont }}^{\text {low }} h_{\text {inter }}^{\text {low }}}, \quad R_{\mathrm{T}}^{\text {high }}=\frac{1}{\pi d_{\mathrm{M}} L_{\text {cont }}^{\text {high }} h_{\text {inter }}^{\text {high }}},
$$

where $d_{\mathrm{M}}=(D-d) / \ln (D / d)$ is the log-mean diameter of the DWCNT, and $h_{\text {inter }}$ is the interlayer heat transfer coefficient. For all DWCNTs, $d_{\mathrm{M}} \approx d_{\mathrm{m}}=(D+d) / 2=d+t$, where $t$ is the interlayer spacing.

The thermal resistance of a very short tube (say, whose length is smaller than the intrinsic phonon mean free path, on the order of $100 \mathrm{~nm}^{1}$, leading to a ballistic thermal transport of the tube), is a constant, independent of its length, i.e.,

$$
R_{\mathrm{T}}^{\text {intra }}=\frac{1}{\pi d t h_{\text {inner }}^{\text {ball }}} .
$$

where $d$ is the diameter, $t$ the wall thickness, and $h_{\text {inner }}^{\text {ball }}$ the ballistic heat transfer coefficient of the inner tube.

To determine $h_{\text {inter }}$ and $h_{\text {inner }}^{\text {ball }}$, we performed MD simulations and found that in the $(5,5)(10,10)$ device at $300-500 \mathrm{~K}$ temperature difference $h_{\text {inter }}^{\text {low }}=9.41 \times 10^{6} \mathrm{Wm}^{-2} \mathrm{~K}^{-1}, \quad h_{\text {inter }}^{\text {high }}=7.68 \times 10^{6} \mathrm{Wm}^{-2} \mathrm{~K}^{-1}$, and $h_{\text {inner }}^{\text {ball }}=3.53 \times 10^{9} \mathrm{Wm}^{-2} \mathrm{~K}^{-1}$. Using these parameters, the temperature gradient on the inner tube, as well as the local temperature of the inner tube can be determined. 
Gradient force. From a previous analytical model ${ }^{2}$, the gradient force on the inner tube, i.e., the reaction force on the outer tube caused by the gradient on the inner tube, can be expressed as

$$
F_{\mathrm{g}}=\frac{\eta^{\text {in }} \pi D L_{\text {cont }}}{A_{0}} \nabla T
$$

where $\eta^{\text {in }}=5.33 \mu \mathrm{eV} /$ atom $/ \mathrm{K}$ is a potential coefficient between two fully contacting graphene layers, $D$ the outer tube diameter, $L_{\mathrm{cont}}$ the contact length, $A_{0}$ the area per atom, and $\nabla T$ the temperature gradient along the inner tube. However, this equation does not include the effect of the tube curvature because it is derived through a model of two contact graphene layers. To account the effect of the tube curvature, we modify the equation as

$$
F_{\mathrm{g}}=\frac{\eta^{\mathrm{in}} \pi d_{\mathrm{m}} L_{\mathrm{cont}}}{A_{0}} \nabla T
$$

in which $d_{\mathrm{m}}$ is the mean diameter of the inner and outer tubes. Using the above obtained temperature gradient, we have

$$
F_{\mathrm{g}}=\frac{\eta^{\mathrm{in}} \pi d_{\mathrm{m}}\left(L_{\text {cont }}^{\text {low }}+L_{\text {cont }}^{\text {high }}\right)}{A_{0}} \frac{1}{1+d t h_{\text {inner }}^{\text {ball }}\left(\frac{1}{d_{\mathrm{m}} L_{\text {cont }}^{\text {low }} h_{\text {inter }}^{\text {low }}}+\frac{1}{d_{\mathrm{m}} L_{\text {cont }}^{\text {high }} h_{\text {inter }}^{\text {high }}}\right)} \frac{\Delta T}{\delta+\left(L_{\text {cont }}^{\text {low }}+L_{\text {cont }}^{\text {high }}\right) / 2} .
$$

In Model II devices, the outer tubes are always in full contact with the inner tube no matter where the inner tube is located. The contact lengths are thus equal to the length of the outer tube, i.e., $L_{\mathrm{cont}}^{\text {low }}=L_{\mathrm{cont}}^{\text {high }}=L$. The gradient force can therefore be written as

$$
F_{\mathrm{g}}^{\mathrm{II}}=\frac{2 \eta^{\text {in }} \pi d_{\mathrm{m}} L}{A_{0}} \frac{1}{1+\frac{d t h_{\text {inner }}^{\text {ball }}}{d_{\mathrm{m}} L}\left(\frac{1}{h_{\text {inter }}^{\text {low }}}+\frac{1}{h_{\text {inter }}^{\text {high }}}\right)} \frac{\Delta T}{\delta+L} .
$$

However, in Model I devices, the contact length between the outer tube and the inner tube varies with the position of the inner tube. When the inner tube is at the center of the two outer tubes, we have $L_{\text {cont }}^{\text {low }}=L_{\text {cont }}^{\text {high }}=(l-\delta) / 2$. The initial gradient force can thus be calculated by

$$
F_{\mathrm{g}}^{\mathrm{I}}=\frac{2 \eta^{\text {in }} \pi d_{\mathrm{m}}(l-\delta)}{A_{0}} \frac{1}{1+\frac{d t h_{\text {inner }}^{\text {ball }}}{d_{\mathrm{m}}(l-\delta) / 2}\left(\frac{1}{h_{\text {inter }}^{\text {low }}}+\frac{1}{h_{\text {inter }}^{\text {high }}}\right)} \frac{\Delta T}{(\delta+l) / 2} .
$$


The gradient forces in the considered Model I and Model II cases can then be theoretically estimated as $F_{\mathrm{g}}^{\mathrm{I}} \approx \frac{41.78(l-\delta)^{2}}{(378 \mathrm{~nm}+l-\delta)(l+\delta)} \mathrm{pN}$, and $F_{\mathrm{g}}^{\mathrm{II}} \approx \frac{41.78 L^{2}}{(189 \mathrm{~nm}+L)(L+\delta)} \mathrm{pN}$, respectively, by taking $\Delta T=200 \mathrm{~K}$.

Edge force. Similar to the gradient force, the edge force can be calculated by ${ }^{2}$

$$
F_{\mathrm{e}}=\frac{\left(\eta^{\mathrm{edge}}-\eta^{\mathrm{in}}\right) \pi d_{\mathrm{m}}}{A_{0}} T
$$

where $\eta^{\text {edge }}=7.29 \mu \mathrm{eV} /$ atom $/ \mathrm{K}$ is a potential coefficient between edge atoms of a graphene layer and another contacting graphene layers, $T$ the local temperature of the outer tube in contact with the end of the inner tube, and the mean diameter $d_{\mathrm{m}}$ instead of the outer tube diameter $D$ is again used to account the curvature effect that is not included in the original equation.

In the considered Model I device, the edge forces acting on the inner tube ends can thus be estimated as $F_{\mathrm{e}}^{\text {low }}=11.3 \mathrm{pN}$ and $F_{\mathrm{e}}^{\text {high }}=18.9 \mathrm{pN}$ for the ends in contact with the low $(300 \mathrm{~K})$ and high $(500 \mathrm{~K})$ temperature outer tubes, respectively. We note that the theoretically predicted value is slightly larger than the MD calculated results (Figure 6c, and data are listed in Table S1) because here the effect of the inner tube temperature is not included.

Table S1. The edge force from molecular dynamics simulations for different combinations of temperatures of the inner and outer tubes.

\begin{tabular}{|c|c|c|c|c|c|c|}
\hline \multirow{2}{*}{\multicolumn{2}{|c|}{ Edge force $(\mathrm{pN})$}} & \multicolumn{5}{|c|}{ Outer Tube Temperature (K) } \\
\hline & & 100 & 200 & 300 & 400 & 500 \\
\hline \multirow{5}{*}{ 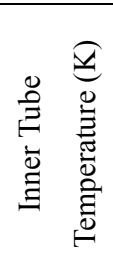 } & 100 & 2.90 & 5.37 & 7.86 & 10.18 & 12.90 \\
\hline & 200 & 2.70 & 5.12 & 7.38 & 9.60 & 12.78 \\
\hline & 300 & 2.30 & 4.67 & 7.34 & 9.84 & 12.73 \\
\hline & 400 & 1.92 & 4.28 & 6.81 & 8.90 & 11.94 \\
\hline & 500 & 1.70 & 3.60 & 6.65 & 9.15 & 11.41 \\
\hline
\end{tabular}

Attraction force. The attraction force depends not only on the geometry of the device, but also on the temperatures of the inner and outer tubes. It is thus difficult to derive an explicit expression to account for all these factors, and no explicit expression is to date available for the temperature dependent attraction 
force. To get a straightforward expression for the attraction force, we performed detailed MD calculations and found that the attraction force is linearly dependent on both the temperatures of the inner and outer tubes. By fitting the MD results (Figure 6b, and data are listed in Table S2), we can obtain an empirical expression for the temperature dependent attraction force,

$$
F_{\mathrm{a}}=F_{0}-\alpha^{\text {in }} T_{\text {inner }}-\alpha^{\text {out }} T_{\text {outer }} .
$$

For $(5,5) /(10,10)$ devices, we have $F_{0}=910 \mathrm{pN}, \alpha^{\text {in }}=0.0734 \mathrm{pN} / \mathrm{K}$ and $\alpha^{\text {out }}=0.0391 \mathrm{pN} / \mathrm{K}$ where $T_{\text {inner }}$ and $T_{\text {outer }}$ are the temperatures of the inner and outer tubes, respectively.

Table S2. The attraction force from molecular dynamics simulations for different combinations of temperatures of the inner $(5,5)$ and outer $(10,10)$ tubes.

\begin{tabular}{|c|c|c|c|c|c|c|c|}
\hline \multirow{2}{*}{\multicolumn{3}{|c|}{$\begin{array}{l}\text { Attraction force } \\
\qquad(\mathrm{pN})\end{array}$}} & \multicolumn{5}{|c|}{ Outer Tube Temperature (K) } \\
\hline & & & 100 & 200 & 300 & 400 & 500 \\
\hline \multirow{5}{*}{$\begin{array}{l}\stackrel{8}{ٍ} \\
\stackrel{\Xi}{\Xi} \\
\stackrel{\Xi}{\Xi}\end{array}$} & \multirow{5}{*}{ 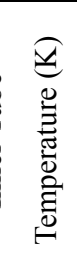 } & 100 & 898.82 & 894.13 & 889.86 & 885.93 & 881.84 \\
\hline & & 200 & 890.97 & 887.87 & 883.46 & 879.01 & 875.00 \\
\hline & & 300 & 883.88 & 879.69 & 876.15 & 872.17 & 868.12 \\
\hline & & 400 & 876.17 & 872.38 & 869.04 & 864.21 & 860.95 \\
\hline & & 500 & 868.24 & 863.95 & 860.32 & 857.15 & 853.83 \\
\hline
\end{tabular}

Total driving force. For the device we considered in Figure 7, the initial driving force can be presented by superposing the above obtained force components. When the distance between the outer tubes is short (say, less than $l-2 L=60 \mathrm{~nm}$ ), the driving force for the inner tube is given by

$$
F^{\mathrm{II}}=\sum F_{\mathrm{a}}+\sum F_{\mathrm{g}}=\frac{71.14 L^{2}}{(189 \mathrm{~nm}+L)(L+\delta)},
$$

while when the distance between the outer tubes is long (say, larger than $l-2 L=60 \mathrm{~nm}$ ), the driving force is

$$
F^{\mathrm{I}}=\sum F_{\mathrm{e}}+\sum F_{\mathrm{a}}+\sum F_{\mathrm{g}}=-15.53-\frac{(l-\delta)}{(378 \mathrm{~nm}+l-\delta)(l+\delta)}(29.36 \delta-41.78(l-\delta)) .
$$

\section{References}

1. Pop, E.; Varshney, V.; Roy, A. K. MRS Bulletin 2012, 37, (12), 1273-1281.

2. Guo, Z.; Chang, T.; Guo, X.; Gao, H. Journal of the Mechanics and Physics of Solids 2012, 60, (9), 1676-1687. 